\title{
$\begin{array}{llllllllll}\text { B } & 0 & 0 & K & \text { R } & \text { E V I E W }\end{array}$
}

\section{Introduction to Color Imaging Science}

Hsien-Che Lee, 695 pages, ISBN 0-52184388-X, Cambridge University Press (2005), \$110 hardbound.

Reviewed by Gaurav Sharma, University of Rochester, Rochester, New York

This book provides an introduction to the scientific principles that govern the operation of color imaging devices and systems. As most readers of this review are well aware, the "science" of color imaging is, in fact, not just a single scientific discipline, but an amalgamation of elements from a number of fields, including, among others, physics, chemistry, optics, and vision. Creating a single book that provides a concise, yet coherent and accurate, description of relevant ingredients is an elusive, if not unattainable, goal. Hsien-Che Lee's book comes amazingly close to fulfilling this objective. The book is comprehensive in its coverage of the primary scientific principles of color imaging. The organization of material in the book follows a logical framework that aids understanding and assimilation of these principles. The writing style is clear and lucid and several chapters include useful insights and details that are rarely covered in other books on this topic. These characteristics combine to make the book informative as well as enjoyable reading.

The book should prove invaluable to its target audience-the increasing number of scientists and engineers who are involved directly or indirectly in the design and use of color imaging systems. In addition, it will also be useful as a text for graduate level courses and as a self-study guide for students preparing for careers in this area. The material in the book should be largely accessible to most readers that possess an undergraduate degree in the sciences or engineering. Given the wide swath that the book covers, most readers will, on occasion, find it necessary to refresh/extend their background knowledge in order to follow the technical details of the exposition. A strong point of the book is that presentations of mathematical expressions and formulas are usually accompanied by text and graphs that communicate the physical intuition behind the equations. This particularly aids the assimilation of concepts for which analytic derivations are either in- tractable or unreasonably long for inclusion.

The contents of the book are organized into 22 closely intertwined chapters. An appendix summarizes commonly used data sets such as color-matching functions, cone fundamentals, and standard illuminants. The citations provided in the chapters, as pointers to literature with additional details on individual topics and related work, amount to a list of more than 1,000 references. The first chapter serves as an introduction to the subject and provides an overview for the book. The remaining chapters are organized into six parts.

The first three parts of the book cover the abstract scientific principles of color imaging that require minimal reference to color devices and technology. Chapters 2 through 8 introduce light and color. This part of the book begins with a short overview of the nature of light as it relates to imaging systems, and then covers key concepts of radiometry, blackbody radiators, the physical theory of light-matter interaction, and principles of photometry and colorimetry. Particularly welcome parts of these chapters are the concise introduction to radiometry and coverage of the bidirectional reflectance distribution function, which are often overlooked in texts on image processing and color imaging. The next three chapters ( 9 through 11), cover optical image formation in imaging systems and in the human eye. These chapters touch upon the geometry of perspective projection, geometrical/physical optics, lens radiometry, aberrations, and imaging distortions. Chapters 12 through 14 cover the physiology and psychophysics of human vision and provide a whirlwind tour of the elements of the human visual system that come into play after the retinal optics going from the retinal mosaic, via the neural pathways, and to the brain. The structure and functions of these elements are highlighted along with visual phenomena and the models used to represent these in color imaging. Chapter 14 presents color order systems that develop a representation of color that conforms to visual perception.

The latter three parts of the book cover principles and techniques that involve color technology devices and evaluation and processing methods that are often closely coupled with these devices. Chapters 15 through 18 present tools for color imaging. This umbrella topic covers color measurement, color device calibration, and tone and color reproduction. This part of the book takes on a more technological flavor as specific algorithms and technologies are described. Color image capture and display devices are covered in Chaps. 19 and 20. Film and digital cameras and scanners are discussed, as are cathode-ray-tube, liquid-crystal, plasma, and electroluminescent displays. Color printing technologies including offset lithography, xerography, and inkjet, along with halftoning and printer calibration techniques are also clubbed together with display devices. Chapters 20 and 21 on image quality and image processing form the last part of the book. Chapter 20 summarizes principles for conducting subjective and objective image quality evaluation. Chapter $21 \mathrm{cov}-$ ers image processing for segmentation, edge detection, and denoising, and highlights how color imaging principles are applicable in these problems, particularly in the choice of color space and vectorial treatment instead of separable processing of color channels.

Early in the book (p. 2) the author states his pedagogical philosophy: "This book is written based on the belief that for a beginning color imaging scientist or engineer, a basic, broad understanding of the physical principles underlying every step in the imaging chain is more useful than an accumulation of knowledge about details of the various techniques." The book adheres to this philosophy throughout. Accordingly, readers can expect to see excellent coverage of principles and should look elsewhere if they are looking primarily for an overview of existing techniques. Overall the exclusion has a positive side effect in increasing the useful life of the book.

The presentation in the first three parts of the book follows a logical progression within each topic, beginning with principles that most graduates in science and engineering are familiar with, and then narrowing the focus and deriving/ summarizing more pragmatic physical models/concepts that are common in color imaging. For example, in Chap. 5, the section on the propagation of light in media begins with Maxwell's equations. The solution thereof for a linear, homogeneous, isotropic material is presented and connected via suitable analysis to the common optical concepts of refraction, dispersion, 
and reflection. The related ideas of scattering, transmission, absorption, and diffraction are briefly described and a summary of the useful mathematical results is included. The theoretical/empirical development for these concepts would be much too long and detailed to interest most readers, and is included only by reference to relevant literature. Subsequently, in Chap. 8 , these are used as the basis for deriving the commonly used Kubelka-Munk model under the two-flux model. Other parts of the book adhere to the same model. The presentation on each topic is shortunderstandably from a desire to have a manageable total book length. Fortunately, the brevity does not come at the expense of precision, which is indeed a remarkable feat for a book covering such diverse ground. Within a chapter, the nature of information and the level at which it is addressed varies significantly among sections addressing different topics. For instance, Chap. 8 includes both detailed analytic sections that develop mathematical models for light interactions with colorants and media and (understandably) terse verbal sections on the physics/chemistry of materials that lead to spectral variation and thereby color. Though this can make the material in a chapter appear rather nonuniform and is occasionally distracting, a reorganization is only likely to compromise readability and comprehension and therefore is not desirable.

The presentation on the latter three parts also attempts to follow the pedagogical philosophy mentioned earlier but is forced to yield in order to relate the presentation to current color devices. Therefore, the book does introduce specific methods and techniques in Chaps. 15 through 19 in order to illustrate some of the design principles. While most parts here are still very well written and develop extensive connections with the general principles presented in the first three parts, for some topics the treatment is quite minimalistic. Particular examples of such topics are: color fidelity in capture devices, demosaicking, halftoning in Chaps. 18 and 19 , and the rather sparse sampling of image processing in Chap. 21. This is understandable in view of the book length constraints but the imbalance is rather striking given the comprehensiveness of the first three parts. Perhaps presentation of the limited examples as case studies might have been preferable.

A comparison of the book with other texts in this area is also instructive. The first chapter includes an extensive categorized list of other books in the areas of color science, color imaging, and optics. Some of these cover specific subjects included in the book, albeit in greater detail, and others focus on subject areas different from the book. The closest book in terms of content is probably the book by Hunt, which is, however, still quite different. The present book is more rigorous in its coverage than Hunt's book, covers more science (particularly in optics, radiometry, and image formation) and less technology (e.g., film and hardcopy reproduction), and is less vested in film-based color photography. The digital color imaging handbook ${ }^{2}$ is likely the most complementary to the present book, where a significantly abbreviated and shorter introduction to the science is combined with more extensive chapters on algorithms and techniques.

One limitation of the book is the lack of any color plates or figures throughout the book. This is surprising given the main subject area of the book. However, given the focus of the book on principles, this does not excessively detract from its util- ity. It does, probably, make it harder for someone with little experience in color to relate concepts to practice and perception.

For self-study and classroom instruction, the organization of the book provides an excellent structural blueprint. Most chapters include problems that could form the basis of homework assignments. For instructors adopting the book for a course, a solutions manual is available from the publisher. One challenge with using the book for classroom instruction seems to be its large size and monolithic character, which does not seem amenable to partitioning into semester/quarter-size units. Since the author has already used the material in teaching classes, road maps of possible one- or two-semester course sequences from the material in the book would be quite useful.

Overall the book represents an excellent synthesis of the basic scientific principles across multiple disciplines that apply in color imaging. If you currently work on or plan to work on color imaging, this well-organized and thorough tome should occupy a handy spot on your bookshelf.

\section{References}

1. R. W. G. Hunt, The Reproduction of $\mathrm{Co}-$ lour, 6th ed., John Wiley and Sons, West Sussex, England (2004).

2. G. Sharma, Ed., Digital Color Imaging Handbook, CRC Press, Boca Raton, Florida (2003).

Gaurav Sharma is an associate professor in the Electrical and Computer Engineering Department at the University of Rochester, Rochester, New York. He also holds a courtesy appointment in the Department of Biostatistics and Computational Biology within the same university. His research interests include color science and imaging, multimedia security, visual sensor networks, and genomic signal processing. 